\title{
Diversidad genética de Handroanthus heptaphyllus a partir de calidad fisiológica de semillas
}

\author{
Genetic diversity of Handroanthus heptaphyllus from seed physiological quality
}

\author{
Tamara Mariel Valdovinos ${ }^{a *}$, Rinaldo Cesar de Paula ${ }^{b}$ \\ *Autor de correspondencia: ${ }^{a}$ Universidade Estadual Paulista, Faculdade de Ciências Agrárias e Veterinárias (UNESP-FCAV), \\ Programa de Pos-graduação en Agronomia (Genética e Melhoramento de Plantas), Jaboticabal, São Paulo, Brasil, \\ tel.: +55 16-999613883, ta.mariel@hotmail.com \\ ${ }^{\mathrm{b}}$ Universidade Estadual Paulista, UNESP-FCAV, Jaboticabal, San Pablo, Brasil.
}

\begin{abstract}
SUMMARY
Handroanthus heptaphyllus (lapacho negro) is one species that stands out for its high economic value, mainly for the high quality of its wood; it is also required for medicinal use, landscaping and urban forestry projects. This study aimed at determining the genetic diversity among $H$. heptaphyllus mother trees from traits of seed physiological quality, evaluated in tests of germination and vigor; and to identify the most influential variables for genetic diversity among trees. The seeds were collected from 25 mother trees, located in the city of Jaboticabal, Sao Paulo, Brazil. In total, 11 traits of physiological quality of seeds, determined by germination, electrical conductivity, accelerated aging and submersion tests, were evaluated. Five replications of 20 seeds were used for each mother tree evaluated. Genetic diversity was assessed using the hierarchical method of Ward and dispersion by Principal Components from the Euclidean Distance calculated between pairs of access. Genetic diversity among the mother trees was expressive. The peak value and electric conductivity were the most effective traits for differentiating trees. The distribution of 25 mother trees in the bi-dimensional plane, from the analysis of the main components, detected the similarities and differences among the trees, based on the evaluated traits, and was consistent with the clusters formed with the Ward's method. The peak value, synchronization index and seedlings length were the variables with higher diversity. This result allows the identification of mother trees for seed collection, supporting conservation programs and genetic improvement of this species.
\end{abstract}

Key words: germination and vigor, clusters analysis, breeding, conservation.

\section{RESUMEN}

Handroanthus heptaphyllus (lapacho negro) es una especie arbórea de alto valor económico, principalmente por la calidad de su madera; además tiene uso medicinal y en proyectos paisajísticos para arborización urbana. El presente trabajo tuvo como objetivos determinar la variabilidad genética entre árboles de H. heptaphyllus a partir de caracteres de calidad fisiológica de semillas, evaluados en ensayos de germinación y vigor; e identificar las variables más influyentes para la diversidad genética entre árboles. Las semillas fueron recolectadas de 25 árboles madre, localizados en la ciudad de Jaboticabal, San Paulo, Brasil. Se evaluaron 11 caracteres de calidad fisiológica de semillas, mediante ensayos de germinación, conductividad eléctrica, envejecimiento acelerado e inmersión. Se utilizaron cinco repeticiones de 20 semillas por cada árbol madre. La diversidad genética se evaluó utilizando el método jerárquico de Ward y la dispersión por componentes principales, a partir de la distancia euclidiana calculada entre los pares de accesos. La variabilidad genética entre los árboles resultó expresiva. El valor pico y la conductividad eléctrica fueron las características más efectivas para la diferenciación de los árboles. El análisis de componentes principales detectó semejanzas y diferencias entre los árboles, basadas en las características evaluadas, y se mostró coherente con los agrupamientos del método de Ward. El valor pico de la germinación, el índice de sincronización del proceso germinativo y la longitud de las plántulas fueron las que presentan mayor diversidad. Estos resultados permiten identificar árboles para la recolección de semillas, dando soporte a programas de conservación y mejoramiento genético de la especie.

Palabras clave: germinación y vigor, análisis de agrupamiento, mejoramiento, conservación.

\section{INTRODUCCIÓN}

Handroanthus heptaphyllus (Mart.) Mattos (lapacho negro) es una especie arbórea de la familia Bignoniaceae, de alto valor económico. Además, se destaca su importan- cia ecológica y sus propiedades medicinales. Su madera de alta calidad es utilizada para la construcción civil y naval. Bastante común en proyectos de paisajismo y arborización urbana, debido a su intensa floración de color rosa (Carvalho 2003). Por causa de su crecimiento relativamente rápi- 
do, es indicada para sistemas agroforestales y silvicultura industrial (CNCFlora 2012). Es una especie que se adapta a diversos tipos de suelo y clima (Tonetto et al. 2015) y con distribución natural en Brasil, Uruguay (Grings y Brack 2011), Paraguay, Argentina y Bolívia (Gentry 1992). Informaciones sobre manejo y/o análisis de semillas de esta especie son necesarias, para que puedan ser caracterizados sus atributos fisiológicos y su variabilidad genética.

La obtención de semillas de especies forestales constituye una parte indisociable del proceso de producción de plantas, y esta producción tiene diversas finalidades y aplicaciones, como ser, conservación ex situ por medio del establecimiento y la manutención de bancos de germoplasma, investigación científica, mejoramiento de especies nativas para uso comercial o extractivo, prácticas de restauración ecológica, paisajismo, arborización urbana y uso ornamental (Martins 2012).

Es fundamental utilizar lotes de semillas genéticamente diversos, para proveer suficiente variación, y que sean adaptadas a la ecología de las diferentes regiones (Broadhurst et al. 2006), sobre todo, considerando que el potencial adaptativo y evolutivo de las poblaciones, es dependiente, en gran medida, del nivel de variabilidad genética (Ribeiro y Rodrigues 2006). Por otro lado, a menudo, se desconoce la estructura genética de las poblaciones que son fuentes de semillas (Martins 2012). La falta de atención, en cuanto a la base genética, puede provocar el llamado "efecto fundador" y pérdida de diversidad por deriva genética, en poblaciones establecidas por regeneración artificial (Giudice-Neto et al. 2005).

La calidad fisiológica de las semillas puede ser definida por dos características diferentes: la germinación y el vigor de semillas (Labouriau 1983, Marcos Filho 1999). La germinación, evaluada en ensayos de germinación, busca determinar el máximo potencial germinativo de un lote de semillas, bajo condiciones medio ambientales consideradas óptimas. El vigor de semillas, coloca en evidencia, algunos atributos más sutiles de la calidad fisiológica, los cuales no logran ser detectados en los ensayos de germinación, por consiguiente, el vigor es determinado en condiciones ambientales desfavorables o bajo la deficiencia de alguna función bioquímica o fisiológica. Por consiguiente, los ensayos de vigor son usados en distintas etapas de los programas de producción de semillas, siendo útiles para evaluar el potencial fisiológico de lotes con germinación semejante, seleccionar lotes para siembra, determinar la calidad fisiológica y como auxilio para los métodos de selección durante el mejoramiento de plantas, entre otras (Marcos Filho 1999).

El conocimiento de la diversidad, en las características fisiológicas de las semillas, contribuye al uso adecuado de las mismas, como ejemplo, ayuda a definir las estrategias de recolección, principalmente la selección de árboles madre productores de semillas. Además de contribuir a la conservación y al mejoramiento genético de las especies (Roveri Neto y Paula 2017).
La hipótesis de este trabajo es que existe variabilidad genética entre árboles de $H$. heptaphyllus y que es posible evaluarla por medio de características de las semillas. A base de lo expuesto y a la creciente demanda por semillas en cantidad y calidad adecuada, el objetivo de este estudio es determinar la variabilidad genética entre árboles madre de H. heptaphyllus a partir de caracteres de calidad fisiológica de semillas, e identificar los caracteres más influyentes para la diversidad genética entre los árboles.

\section{MÉTODOS}

Para el desarrollo de este trabajo fueron utilizadas semillas de $H$. heptaphyllus provenientes de frutos en inicio de dehiscencia, que mostraban las primeras aperturas, pero todavía sin presentar dispersión de semillas. Los frutos fueron recolectados de 25 árboles, conformados por árboles remanentes y plantados, localizados en la Facultad de Ciencias Agrarias y Veterinarias de la Universidad Estadual Paulista (FCAV-UNESP), Campus de Jaboticabal, y en áreas urbanas de la ciudad de Jaboticabal, en septiembre del 2014. La selección de los árboles se basó en la presencia abundante de frutos y se respetó una distancia de $20 \mathrm{~m}$ entre los árboles seleccionados, esto para disminuir la posibilidad de cruzamientos relacionados. Sin embargo, considerando el sistema de cruzamiento de $H$. heptaphyllus, en que, debido a la ocurrencia de cruzamientos correlacionados, ocurre un aumento de la coancestría dentro de las progenies, Mori et al. (2015) recomiendan, para fines de mejoramiento, conservación o recuperación de áreas degradadas, recolectar semillas de, al menos, 49 árboles, para obtener un tamaño efectivo $(\mathrm{Ne})$ de 150.

Los frutos fueron recolectados directamente de los árboles, con ayuda de una podadora aérea. Enseguida se colocaron dentro de fundas plásticas, y fueron llevadas al Vivero Experimental de Plantas Ornamentales y Forestales de la FCAV-UNESP, donde pasaron por un proceso de secado natural. Luego de completarse la abertura natural de los frutos, las semillas fueron extraídas de forma manual, colocadas en bolsas de papel, debidamente identificadas e inmediatamente llevadas para el Laboratorio de Semillas y Mejoramiento Forestal, de la FCAV-UNESP, donde fueron beneficiadas y utilizadas para la realización de los ensayos de calidad fisiológica (germinación y vigor).

Para analizar la germinación, las semillas de cada árbol fueron colocadas en cajas de plástico transparente, de $11 \mathrm{x}$ $11 \times 3,5 \mathrm{~cm}$, con tapa, sobre dos hojas de papel filtro humedecidas con agua destilada (volumen de agua equivalente a 2,5 veces el peso del papel), siguiendo las recomendaciones de las Reglas para Análisis de Semillas (Brasil 2009). El experimento de germinación fue realizado en cámaras verticales, tipo B.O.D., a $25^{\circ} \mathrm{C}$ y fotoperiodo de 8 h. Se registró diariamente el número de semillas germinadas, $\mathrm{y}$ se adoptó como criterio de germinación, a la formación de raíz primaria con largo igual o mayor a $0,5 \mathrm{~cm}$, para el cálculo del índice de velocidad de germinación (IVG). Al 
final de este ensayo, que tuvo una duración de 21 días, fueron calculados: porcentaje de germinación $(\mathrm{G})$; índice de velocidad de germinación (IVG), según Maguire (1962); valor pico de la germinación (VP) -que corresponde al valor máximo de la razón entre el porcentaje de germinación acumulada y el número de días necesarios para alcanzarla (Czabator 1962); valor germinativo (VG) -resultado del producto entre el valor pico y la germinación media diaria (GMD - razón entre el total de semillas germinadas y el número total de días (duración) del ensayo de germinación), conforme Czabator (1962) y el índice de sincronización (E) utilizando la siguiente expresión (Labouriau 1983):

$$
\bar{E}=-\sum_{i=1}^{k} f_{i} \cdot \log _{2}\left(f_{i}\right) \text { siendo } f_{i}=\frac{n_{i}}{\sum_{i=1}^{k} n_{i}}
$$

Donde $\mathrm{f}_{\mathrm{i}}$ : frecuencia relativa de la germinación; k: último día de observación y $\mathrm{n}_{\mathrm{i}}$ : número de semillas germinadas en el día i.

Al final del ensayo de germinación se determinó el porcentaje de plántulas normales $(\mathrm{PN})$ y anormales $(\mathrm{PA})$ - relación entre el número de plántulas con y sin las estructuras esenciales perfectas, respectivamente, y el número de semillas colocadas para germinar; y también se midió la longitud de plántulas $(\mathrm{CP}, \mathrm{mm})$ en todas las plántulas normales de cada repetición/tratamiento.

Para el ensayo de envejecimiento acelerado (GEA) se utilizaron cajas de plástico transparente ( $11 \times 11$ x 3,5 $\mathrm{cm}$ ), con tapa, conforme el método "gerbox" descrito por Marcos Filho (1999), con $40 \mathrm{~mL}$ de agua destilada en su interior. Las semillas se colocaron encima de una tela de aluminio, formando una camada uniforme, y la tela fue acomodada dentro de las cajas, encima del nivel del agua. Las cajas tapadas fueron colocadas en germinadores verticales tipo B.O.D. regulados a $45{ }^{\circ} \mathrm{C}$, por $72 \mathrm{~h}$ (tiempo determinado en ensayos preliminares, conducidos por 24 , 48,72 y $96 \mathrm{~h}$ ). Una vez transcurridas las $72 \mathrm{~h}$, las muestras de semillas fueron colocadas para germinar bajo las mismas condiciones descritas anteriormente, y luego de 14 días, se registró el porcentaje de germinación (GEA) de las semillas envejecidas.

El experimento de conductividad eléctrica (CE) se realizó a $25^{\circ} \mathrm{C}$, utilizando cinco repeticiones de 20 semillas por árbol madre, con las semillas inmersas en $75 \mathrm{~mL}$ de agua destilada $\left(\leq 2,5 \mu \mathrm{S} \mathrm{cm}^{-1}\right.$ de conductividad) durante 48 $\mathrm{h}$ (tiempo determinado en ensayos preliminares, en que se evaluaron diferentes períodos de inmersión por $24,48,72$, 96 y 120 h). La conductividad eléctrica de la solución fue medida usando un conductímetro de mesa, con constante 1,0 . Se pesaron todas las repeticiones en una balanza analítica $(0,0001 \mathrm{~g})$. Los resultados de la lectura de la conductividad fueron divididos por los respectivos valores de masa fresca $(\mathrm{g})$ de semillas, y los valores fueron expresados en $\mu \mathrm{S} \mathrm{cm} \mathrm{cm}^{-1} \mathrm{~g}^{-1} \mathrm{de}$ semillas.

Para el ensayo de inmersión (GTS) se utilizaron las semillas provenientes del ensayo de conductividad eléctrica, donde estas semillas permanecieron sumergidas en $75 \mathrm{~mL}$ de agua destilada a $25^{\circ} \mathrm{C}$, durante $120 \mathrm{~h}$. Transcurrido este tiempo, las semillas fueron retiradas del agua y colocadas para germinar conforme a procedimientos descritos anteriormente. Luego de 14 días, contados desde el inicio del experimento de germinación, se registró el porcentaje de semillas con desarrollo de raíz primaria (GTS).

El diseño experimental utilizado fue bloques completos al azar con 25 tratamientos (árboles madre) y cinco repeticiones de 20 semillas. Los datos se sometieron a la prueba de normalidad de Lilliefors (Cruz 2001). Y como siete características no alcanzaron la normalidad, se aplicó la transformación logarítmica $[\log (\mathrm{x}+1)]$ en los valores de velocidad de germinación, valor pico y valor germinativo, y la transformación raíz cuadrada $[\sqrt{X+0,5}]$ en los valores de porcentaje de plántulas normales y anormales, y germinación de las semillas envejecidas y sumergidas; donde $\mathrm{X}$ representa las respectivas características, sin embargo, en los cuadros de resultados están presentados los datos originales (sin transformar), para facilitar la interpretación de los mismos. También se realizó la prueba de Bartlett para verificar la homogeneidad entre las varianzas. Para verificar la existencia de variabilidad genética entre los árboles estudiados, con los valores de las variables se realizó un análisis de varianza y las medias fueron agrupadas por la prueba de Scott y Knott a $5 \%$ de probabilidad. Adicionalmente se estimaron los coeficientes de correlación de Pearson entre todas las características evaluadas en los diferentes ensayos.

Para agrupar los árboles madre más similares e identificar los principales caracteres para la divergencia genética, se realizó un análisis de los componentes principales y un agrupamiento usando el método jerárquico de Ward. Para el método de Ward, la medida de disimilitud fue la distancia euclidiana (Cruz et al. 2004), calculada en base a las medias estandarizadas de cada carácter evaluado por árbol madre. Se tomó como punto de corte del eje horizontal del dendrograma, a la media de las distancias euclidianas.

La importancia de las características para estudio de la divergencia genética, se determinó mediante el análisis de componentes principales, de esta manera, se estimaron los valores propios (varianza asociada a cada componente principal) a partir de las raíces características de la matriz $\mathrm{R}$ (matriz de correlación) y los vectores propios (conjunto de coeficientes de ponderación de los componentes principales) a partir de los elementos de los vectores característicos correspondientes. Para identificar la importancia relativa de cada variable, se consideró como de menor importancia, a aquellas variables de mayor vector propio (en valor absoluto) a partir del último componente hasta aquel cuyo valor propio no excedía 0,70 , según las recomendaciones de Cruz et al. (2004).

Para los métodos de Ward y componentes principales se utilizó el paquete estadístico STATISTIC (2011), versión 10.0, los demás análisis estadísticos se realizaron con el GENES (Cruz 2001). 


\section{RESULTADOS}

El análisis de varianza de los 25 árboles detectó diferencias estadísticas $(P>0,01)$ en todas las variables (cuadro 1). En lo referente al coeficiente de variación (CV), se encontraron valores entre $12,2 \%$ a $21,6 \%$, presentando mayores coeficientes de variación las variables: índices de sincronización (21,6\%), porcentaje de plántulas normales $(20,5 \%)$ y anormales $(20,5 \%)$. De todas las variables, el valor pico (VP) y la conductividad eléctrica (CE) resultaron más efectivas para diferenciar los 25 árboles madre, ya que formaron siete y seis grupos de medias, respecti-

Cuadro 1. Valores de F, coeficientes de variación experimental (CV), media general (Media) y comparación de medias de porcentaje de germinación $(\mathrm{G})$, índice de velocidad de germinación (IVG), valor pico (VP), valor germinativo (VG), índice de sincronización informacional (E), longitud de plántulas (CP), porcentaje de plántulas normales (PN) y anormales (PA) del ensayo de germinación; porcentaje de germinación del ensayo de inmersión (GTS), envejecimiento acelerado (GEA) y conductividad eléctrica de masa (CE) evaluados en semillas de 25 árboles madre de Handroanthus heptaphyllus.

F values, experimental variation coefficients (CV), overall average (Mean) and comparison of average for percentage of germination (G), germination speed index (IVG), peak value (VP) germination value (VG) and informational entropy (E), seedlings length (CP), percentage of normal seedlings (PN) and abnormal (PA), of germination test; germination percentage in submersion (GTS), accelerated aging test (GEA) and mass electrical conductivity (CE) evaluated in seeds of 25 in mother trees of Handroanthus heptaphyllus.

\begin{tabular}{|c|c|c|c|c|c|c|c|c|c|c|c|}
\hline & G (\%) & IVG & VP & $\mathrm{VG}$ & $\mathrm{E}$ & $\mathrm{CP}(\mathrm{mm})$ & PN (\%) & PA (\%) & GTS (\%) & GEA (\%) & $\mathrm{CE}\left(\mu \mathrm{S}, \mathrm{cm} \mathrm{g}^{-1}\right)$ \\
\hline Valor F & $27,34 * *$ & $18,99 * *$ & $19,08 * *$ & $23,76^{* *}$ & $4,22 * *$ & $3,44 * *$ & $8,78^{* *}$ & $5,73 * *$ & $10,71 * *$ & $19,61 * *$ & $16,06^{* *}$ \\
\hline CV (\%) & 18,6 & 17,8 & 12,9 & 16,8 & 21,6 & 17,1 & 20,5 & 20,5 & 17,7 & 12,2 & 13,2 \\
\hline Media & 49 & 1,63 & 5,58 & 16,24 & 2,13 & 76,42 & 24 & 29 & 26 & 42 & 249,28 \\
\hline Madre & & & & & Medias & & & & & & \\
\hline 1 & $73 a$ & $2,21 b$ & $8,36 \mathrm{~b}$ & $31,01 \mathrm{a}$ & $2,38 \mathrm{a}$ & $93,06 \mathrm{a}$ & $45 \mathrm{a}$ & $32 b$ & $39 \mathrm{~b}$ & $74 \mathrm{a}$ & $180,05 \mathrm{e}$ \\
\hline 2 & $27 \mathrm{~d}$ & $1,10 \mathrm{c}$ & $3,23 \mathrm{e}$ & $4,95 \mathrm{~d}$ & $1,79 b$ & $81,54 \mathrm{a}$ & $20 \mathrm{c}$ & $15 \mathrm{c}$ & $15 \mathrm{c}$ & $22 d$ & $303,62 c$ \\
\hline 3 & $78 \mathrm{a}$ & $2,58 \mathrm{a}$ & $8,78 b$ & $34,24 a$ & $2,58 \mathrm{a}$ & $76,24 a$ & $42 \mathrm{a}$ & $32 b$ & $48 \mathrm{a}$ & $65 a$ & $184,30 \mathrm{e}$ \\
\hline 4 & $75 \mathrm{a}$ & $3,00 \mathrm{a}$ & $10,95 \mathrm{a}$ & $39,96 a$ & $2,21 \mathrm{a}$ & $82,46 a$ & $35 b$ & $41 \mathrm{a}$ & $34 \mathrm{~b}$ & $53 b$ & $249,18 d$ \\
\hline 5 & $75 a$ & $3,00 \mathrm{a}$ & $10,38 \mathrm{a}$ & $37,17 \mathrm{a}$ & $2,13 \mathrm{a}$ & $83,68 \mathrm{a}$ & $51 \mathrm{a}$ & $26 b$ & $53 a$ & $75 a$ & $133,79 f$ \\
\hline 6 & $47 \mathrm{c}$ & $1,31 \mathrm{c}$ & $4,55 d$ & $10,59 b$ & $2,30 \mathrm{a}$ & $67,48 b$ & $26 b$ & $27 \mathrm{~b}$ & $17 \mathrm{c}$ & $49 b$ & $263,78 \mathrm{c}$ \\
\hline 7 & $33 \mathrm{c}$ & $1,09 \mathrm{c}$ & $3,58 \mathrm{e}$ & $6,35 c$ & $2,06 a$ & $74,32 \mathrm{a}$ & $18 \mathrm{c}$ & $19 \mathrm{c}$ & $17 \mathrm{c}$ & $33 c$ & $266,86 \mathrm{c}$ \\
\hline 8 & $23 d$ & $0,71 d$ & $2,69 f$ & $3,19 \mathrm{~d}$ & $1,61 b$ & $46,86 \mathrm{c}$ & $9 d$ & $20 \mathrm{c}$ & $16 \mathrm{c}$ & $24 d$ & $306,87 \mathrm{c}$ \\
\hline 9 & $51 \mathrm{~b}$ & $1,66 \mathrm{~b}$ & $5,49 \mathrm{c}$ & $13,45 b$ & $2,47 \mathrm{a}$ & $60,66 \mathrm{c}$ & $25 b$ & $30 \mathrm{~b}$ & $22 \mathrm{c}$ & $32 \mathrm{c}$ & $235,42 d$ \\
\hline 10 & $39 \mathrm{c}$ & $1,30 \mathrm{c}$ & $4,44 d$ & $8,28 \mathrm{c}$ & $2,22 a$ & $56,00 \mathrm{c}$ & $18 \mathrm{c}$ & $29 b$ & $15 \mathrm{c}$ & $20 \mathrm{~d}$ & $283,33 \mathrm{c}$ \\
\hline 11 & $60 \mathrm{~b}$ & $2,22 b$ & $7,57 \mathrm{~b}$ & $22,48 \mathrm{a}$ & $2,25 \mathrm{a}$ & $82,86 a$ & $35 b$ & $31 b$ & $40 \mathrm{a}$ & $67 \mathrm{a}$ & $211,04 d$ \\
\hline 12 & $76 a$ & $2,46 a$ & $7,80 \mathrm{~b}$ & $29,11 \mathrm{a}$ & $2,59 a$ & $83,08 \mathrm{a}$ & $46 a$ & $33 b$ & $37 b$ & $56 \mathrm{~b}$ & $173,23 \mathrm{e}$ \\
\hline 13 & $26 \mathrm{~d}$ & $1,01 \mathrm{c}$ & $3,47 \mathrm{e}$ & $5,91 \mathrm{~d}$ & $1,43 b$ & $81,90 \mathrm{a}$ & $10 \mathrm{~d}$ & $20 \mathrm{c}$ & $17 \mathrm{c}$ & $18 \mathrm{e}$ & $280,39 \mathrm{c}$ \\
\hline 14 & $40 \mathrm{c}$ & $0,97 \mathrm{c}$ & $3,31 \mathrm{e}$ & $6,84 \mathrm{c}$ & $2,16 \mathrm{a}$ & $70,48 b$ & $16 \mathrm{c}$ & $29 b$ & $25 \mathrm{c}$ & $37 \mathrm{c}$ & $253,45 d$ \\
\hline 15 & $60 \mathrm{~b}$ & $1,86 \mathrm{~b}$ & $6,13 \mathrm{c}$ & $17,54 b$ & $2,66 a$ & $69,14 b$ & $25 b$ & $43 a$ & $23 c$ & $43 b$ & $218,42 d$ \\
\hline 16 & $37 \mathrm{c}$ & $0,82 d$ & $2,64 f$ & $4,86 \mathrm{~d}$ & $2,32 \mathrm{a}$ & $89,94 a$ & $11 d$ & $36 b$ & $9 d$ & $31 \mathrm{c}$ & $233,50 \mathrm{~d}$ \\
\hline 17 & $29 d$ & $0,63 \mathrm{~d}$ & $2,26 f$ & $3,23 \mathrm{~d}$ & $1,81 \mathrm{~b}$ & $77,28 \mathrm{a}$ & $16 \mathrm{c}$ & $16 c$ & $22 c$ & $32 \mathrm{c}$ & $236,34 d$ \\
\hline 18 & $16 \mathrm{~d}$ & $0,40 \mathrm{~d}$ & $1,32 \mathrm{~g}$ & $0,97 \mathrm{e}$ & $1,47 b$ & $70,14 b$ & $12 d$ & $11 \mathrm{c}$ & $7 d$ & $22 d$ & $355,75 b$ \\
\hline 19 & $72 a$ & $2,75 \mathrm{a}$ & $10,10 \mathrm{a}$ & $34,98 \mathrm{a}$ & $2,26 \mathrm{a}$ & $82,44 a$ & $35 b$ & $40 \mathrm{a}$ & $35 b$ & $66 a$ & $210,97 d$ \\
\hline 20 & $74 a$ & $2,39 a$ & $7,73 b$ & $27,30 \mathrm{a}$ & $2,47 \mathrm{a}$ & $77,28 \mathrm{a}$ & $22 c$ & $55 \mathrm{a}$ & $28 \mathrm{~b}$ & $61 \mathrm{a}$ & $275,60 \mathrm{c}$ \\
\hline 21 & $69 a$ & $2,51 \mathrm{a}$ & $7,83 b$ & $26,46 a$ & $2,45 \mathrm{a}$ & $77,72 \mathrm{a}$ & $26 \mathrm{~b}$ & $44 a$ & $29 b$ & $46 b$ & $224,62 d$ \\
\hline 22 & $17 d$ & $0,77 \mathrm{~d}$ & $3,04 \mathrm{e}$ & $3,71 \mathrm{~d}$ & $1,38 b$ & $84,60 \mathrm{a}$ & $8 \mathrm{~d}$ & $10 \mathrm{c}$ & $16 \mathrm{c}$ & $14 \mathrm{e}$ & $399,93 \mathrm{a}$ \\
\hline 23 & $24 d$ & $0,76 \mathrm{~d}$ & $3,10 \mathrm{e}$ & $4,27 \mathrm{~d}$ & $1,30 \mathrm{~b}$ & $76,74 a$ & $7 \mathrm{~d}$ & $12 \mathrm{c}$ & $10 \mathrm{~d}$ & $13 \mathrm{e}$ & $314,74 \mathrm{c}$ \\
\hline 24 & $65 b$ & $1,71 b$ & $5,27 \mathrm{c}$ & $16,91 b$ & $2,79 a$ & $72,06 \mathrm{~b}$ & $28 \mathrm{~b}$ & $43 a$ & 45 & $44 \mathrm{~b}$ & $238,84 d$ \\
\hline 25 & $45 \mathrm{c}$ & $1,56 \mathrm{~b}$ & $5,41 \mathrm{c}$ & $12,10 \mathrm{~b}$ & $2,15 \mathrm{a}$ & $92,62 \mathrm{a}$ & $13 \mathrm{~d}$ & $32 b$ & $32 b$ & $44 \mathrm{~b}$ & $197,95 \mathrm{e}$ \\
\hline
\end{tabular}

** $P<0,01$ - valor significativo para la prueba F. Medias seguidas por la misma letra en la columna, no difieren entre sí según la prueba de Scott-Knott $(P>0,05)$. 
vamente. En contraste, el índice de sincronización (E) no discriminó bien los árboles. Las medias de valor pico variaron de 1,32 (madre 18) a 10,95 (madre 4) y las de conductividad eléctrica de 133,79 (madre 5) a 399,93 $\mu \mathrm{S}$ $\mathrm{cm}^{-1} \mathrm{~g}^{-1}$ (madre 22), así, ambas variables mostraron amplia variación. La germinación tras el envejecimiento acelerado (GEA) y el valor germinativo (VG), dividieron los árboles madre en cinco grupos, con medias que variaron de $13 \%$ (madre 23 ) a $75 \%$ (madre 5$)$ y de 0,97 (madre 18) a 39,96 (madre 4), respectivamente. Cuatro variables formaron cuatro grupos de árboles madre: la germinación (G) que varió de $16 \%$ (madre 18) a $78 \%$ (madre 3), índice de velocidad de germinación (IVG) cuyas medias fueron de 0,40 (madre 18) a 3,00 (madre 4), porcentaje de plántulas normales $(\mathrm{PN})$ con variación de $7 \%$ (madre 23) a $51 \%$ (madre 5) y la germinación del ensayo de inmersión (GTS) con medias desde $7 \%$ (madre 18) a $53 \%$ (madre 5). La longitud de plántula $(\mathrm{CP})$ y el porcentaje de plántulas anormales (PA) dividieron los árboles en tres grupos, que variaron de 46,86 mm (madre 8) a 93,06mm (madre 1) y de $10 \%$ (madre 22 ) a $55 \%$ (madre 20 ), respectivamente. Para el índice de sincronización (E), apenas se formaron dos grupos y las medias variaron de 1,30 (madre 23) a 2,79 (madre 24). Aunque las variables germinación $(\mathrm{G})$, índice de velocidad de germinación (IVG), germinación del ensayo de inmersión (GTS) y porcentaje de plántulas normales (PN) separaron los árboles en igual número de grupos, se encontraron alteraciones en la constitución de los grupos.

Para las estimativas de los coeficientes de correlación se encontraron valores de alta magnitud $(r>0,70)$ para la mayoría de los pares de caracteres, con excepción de la longitud de plántulas (cuadro 2). En general, las caracte- rísticas evaluadas en el ensayo de germinación presentaron altas estimativas de los coeficientes de correlación entre sí. Como excepción, las correlaciones entre valor pico e índice de sincronización, y las correlaciones entre la variable plántula anormal con plántula normal y con el ensayo de inmersión, que mostraron estimativas moderadas, de 0,50 a 0,70 . La conductividad eléctrica mostró correlaciones negativas, moderadas a altas, con todas las características de calidad fisiológica de semillas, indicando una relación inversa entre conductividad y vigor de semillas. Se evidenció una alta correlación entre los ensayos de envejecimiento acelerado y de inmersión; además, una correlación moderada a alta, de ambas, con todos los caracteres evaluados en el ensayo de germinación.

Con el método de Ward se identificaron siete grupos de árboles de H. heptaphyllus distribuidos dentro de dos conjuntos mayores (I y II) (figura 1). El conjunto I se integró por los grupos 1 al 5, y el conjunto II por los grupos 6 y 7. Comparativamente al conjunto I, el conjunto II agrupó a los árboles de mayor calidad fisiológica, ya que presentaron medias elevadas para las variables: germinación, índice de velocidad de germinación, valor pico, valor germinativo, porcentaje de plántulas normales y germinación en los ensayos de inmersión y envejecimiento acelerado. Sin embargo, esos árboles se diferenciaron por dos características de desempeño de plántulas: longitud (CP) y desarrollo de plántulas anormales (PA). De ese modo, los árboles del grupo 6 (árboles 24, 15, 21, 20, 19 y 4) se caracterizaron por tener altos porcentajes de plántulas anormales y bajos valores de longitud de plántulas, al contrario de los árboles del grupo 7 (árboles 5, 12, 3, 11 y 1). Dentro del conjunto I, donde fueron agrupados

Cuadro 2. Estimativas de correlaciones entre 11 características de calidad fisiológica de semillas evaluadas en los ensayos de germinación, envejecimiento acelerado, inmersión y conductividad eléctrica, en 25 árboles madre de Handroanthus heptaphyllus.

Estimates of correlations between 11 physiological qualities of seed characteristics evaluated in the germination, accelerated aging, electrical conductivity and submersion tests in 25 mother trees of Handroanthus heptaphyllus.

\begin{tabular}{|c|c|c|c|c|c|c|c|c|c|c|}
\hline & IVG & VP & $\mathrm{VG}$ & $\mathrm{E}$ & PN & PA & GTS & GEA & $\mathrm{CE}$ & $\mathrm{CP}$ \\
\hline G & 0,958 & 0,941 & 0,980 & 0,813 & 0,885 & 0,811 & 0,844 & 0,902 & $-0,780$ & 0,274 \\
\hline IVG & & 0,992 & 0,990 & 0,711 & 0,854 & 0,751 & 0,836 & 0,839 & $-0,719$ & 0,284 \\
\hline VP & & & 0,988 & 0,672 & 0,835 & 0,726 & 0,834 & 0,826 & $-0,707$ & 0,284 \\
\hline VG & & & & 0,764 & 0,865 & 0,788 & 0,849 & 0,870 & $-0,756$ & 0,276 \\
\hline E & & & & & 0,689 & 0,857 & 0,626 & 0,720 & $-0,698$ & 0,041 \\
\hline PN & & & & & & 0,514 & 0,829 & 0,877 & $-0,777$ & 0,249 \\
\hline PA & & & & & & & 0,570 & 0,681 & $-0,605$ & 0,106 \\
\hline GTS & & & & & & & & 0,837 & $-0,769$ & 0,304 \\
\hline GEA & & & & & & & & & $-0,813$ & 0,339 \\
\hline $\mathrm{CE}$ & & & & & & & & & & $-0,331$ \\
\hline
\end{tabular}

G - germinación; IVG - índice de velocidad de germinación; $\mathrm{VP}$ - valor pico; $\mathrm{VG}$ - valor germinativo; E - índice de sincronización de la germinación; PN - plántulas normales; PA - plántulas anormales; GTS - ensayo de inmersión; GEA - envejecimiento acelerado; CE - conductividad eléctrica; CP - longitud de plántulas. 


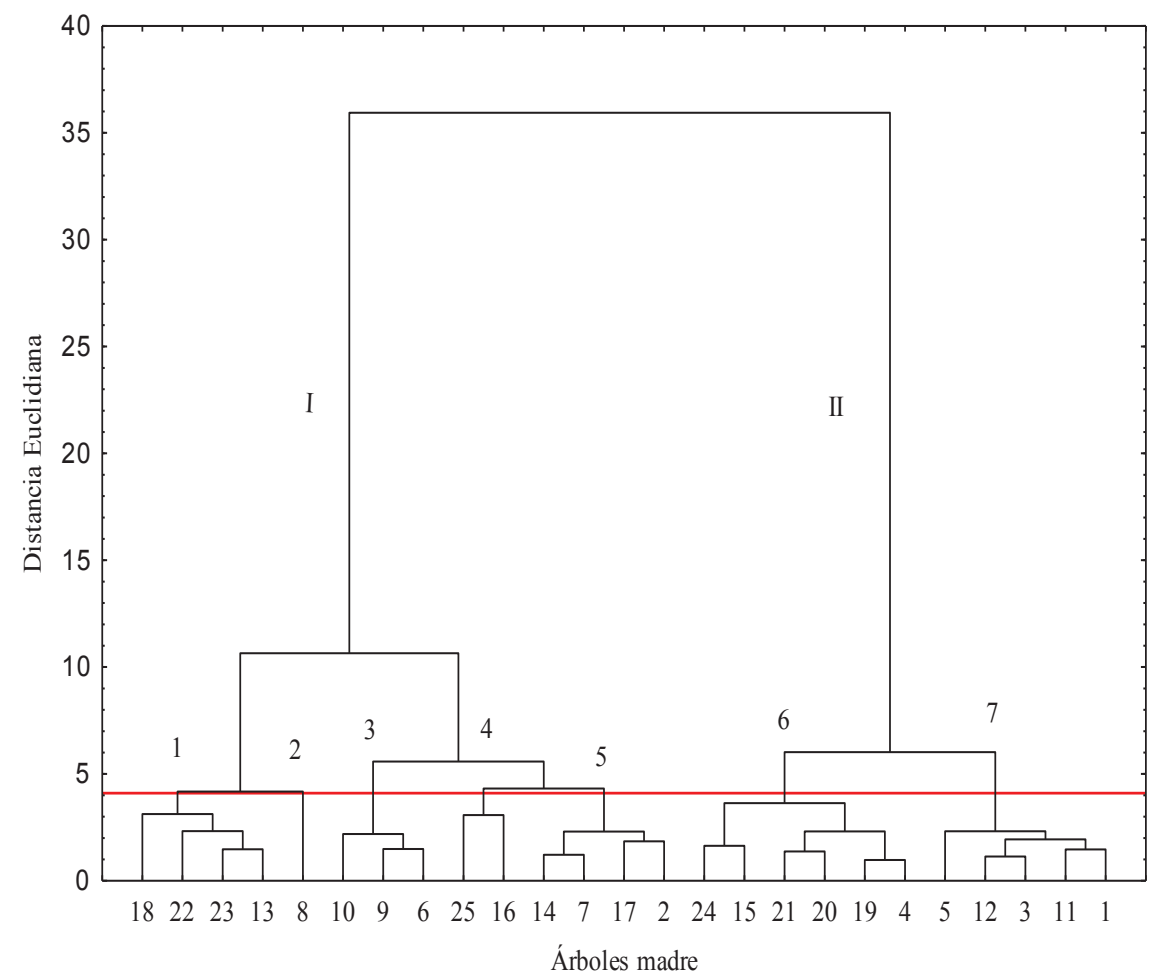

Figura 1. Dendrograma basado en la distancia euclidiana entre 25 árboles madre de Handroanthus heptaphyllus, obtenido por el método Ward a partir de 11 caracteres evaluados en los ensayos de germinación, envejecimiento acelerado, inmersión y conductividad eléctrica de semillas. La línea roja representa el "punto de corte" (definido como la media de las distancias entre pares de árboles madre) de los grupos (identificados con números de 1 a 7 encima de la línea roja) pertenecientes a los conjuntos (I y II).

Dendrogram based on the Euclidean distance between 25 mother trees of Handroanthus heptaphyllus obtained by Ward' method based on 11 characters evaluated in the germination test, accelerated aging, submersion and electrical conductivity of seeds. The red line represents the "point cutting "(defined as the mean of the distances between pairs of mother trees) of the groups (identified with numbers 1 through 7 above the red line) belonging to two conglomerates (I and II).

los árboles con semillas de menor calidad, el grupo 1 se formó con los árboles 18, 22, 23 y 13, el grupo 2 se formó solo con el árbol 8, los árboles 10, 9 y 6 formaron el grupo 3 , los arboles 25 y 16 el grupo 4 y el grupo 5 se formó con los arboles 14, 7, 17 y 2 .

Todos los árboles madre de los grupos 2, 3, 4 y 5, presentan un desempeño germinativo inferior, y algunas diferencias que sobresalen dentro de cada grupo. Los grupos 2 y 3 presentan plántulas de menor longitud, particularmente el árbol madre 8. Los arboles del grupo 4 se distinguen por la longitud de plántulas, aunque tienen altos porcentajes de plántulas anormales y escasas plántulas normales. Los árboles del grupo 5 presentan buen tamaño de plántulas. Entre las características del grupo 1, que son los más contrastantes con el grupo 7, sobresale su baja calidad fisiológica, ya que presentan valores elevados de conductividad eléctrica y menor desempeño germinativo para todas las características evaluadas.

Los dos primeros componentes principales explican $85,3 \%$ de la variación total (cuadro 3; figura 2A).

El primer componente se asoció positivamente con la mayoría de las variables y negativamente con la conducti- vidad eléctrica (figura 2B), y permitió separar a los arboles madre de mejor calidad fisiológica en el cuarto cuadrante (IV) (figura 2A). El segundo componente principal se relacionó positivamente con el índice de sincronización (E) y el porcentaje de plántulas anormales (PA), y de manera negativa con la longitud de plántulas (figura $2 \mathrm{~B}$ ), por tanto, resultó útil para identificar los árboles madre con bajo desempeño germinativo, de forma desordenada en el tiempo, con plántulas anormales y de alturas menores que se ubicaron en el área izquierda e inferior del gráfico (III) (figura 2A).

Al adoptar el criterio citado por Cruz et al. (2004) para identificar los caracteres de menor importancia, es decir, los mayores coeficientes de ponderación en los componentes con valores propios inferiores a 0,70 (cuadro 3, valores subrayados), se evidenció que $14,7 \%$ de la variabilidad contenida en los datos, se concentró entre el tercero y el último componente principal, situación en que ocho, o sea, $73 \%$ de los caracteres resultaron aptos para ser descartados. De acuerdo con estas consideraciones, se determinó eliminar las siguientes variables, en orden de menor importancia: valor germinativo, índice de velocidad de ger- 

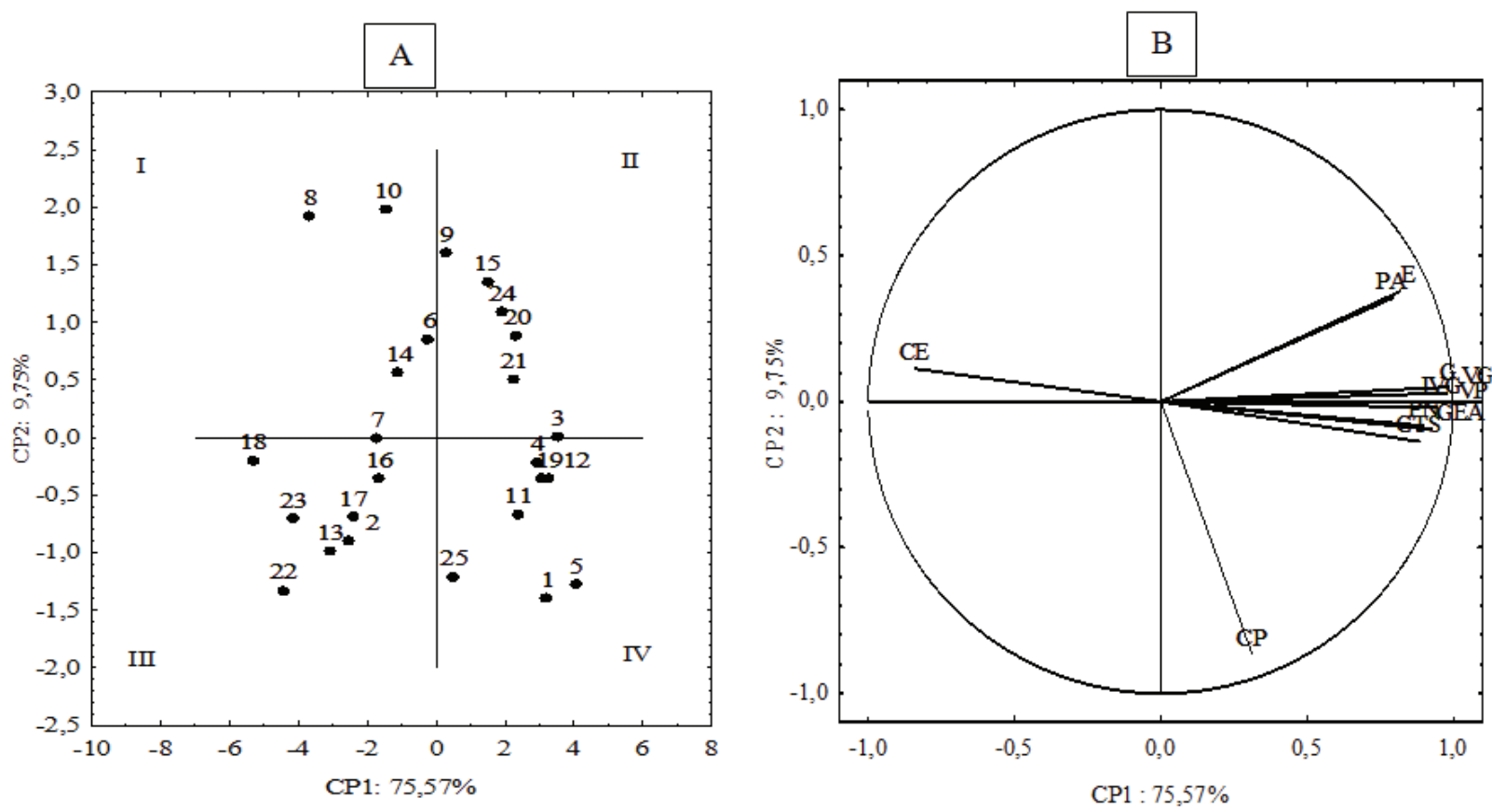

Figura 2. (A) Dispersión grafica de los 25 árboles madre de Handroanthus heptaphyllus en relación al primero y al segundo componente principal, establecidos por la combinación lineal de 11 caracteres de calidad de semillas. (B) Correlación de los 11 caracteres que discriminan la posición de los árboles madre, referente a los dos componentes principales (CP1 x CP2).

(A) Graphic dispersion of 25 mother trees of Handroanthus heptaphyllus in relation to the first and the second main component established by linear combination of 11 characters of seed quality. (B) Correlations of 11 characters which discriminate the position of the mother trees, referring to the two principal components (CP1x CP2).

Cuadro 3. Valores propios (varianza-v) asociados a los componentes principales (CP) y los respectivos vectores propios para los 11 caracteres evaluados en 25 árboles madre de Handroanthus heptaphyllus.

Eigenvalues (variance-v) associated with the principal components (CP) and their eigenvectors for the 11 traits evaluated in 25 mother trees of Handroanthus heptaphyllus.

\begin{tabular}{lllllllllllllll}
\hline \multirow{2}{*}{ CP } & A & AV & \multicolumn{10}{c}{ Autovector asociado } \\
\cline { 5 - 13 } & & & G & IVG & VP & VG & E & PN & PA & GTS & GEA & CE & CP \\
\hline CP1 & 8,313 & 75,575 & 0,342 & 0,333 & 0,328 & 0,340 & 0,284 & 0,312 & 0,276 & 0,308 & 0,322 & $-0,292$ & 0,108 \\
CP2 & 1,072 & 85,325 & 0,049 & $-0,001$ & $-0,020$ & 0,028 & 0,365 & $-0,080$ & 0,344 & $-0,133$ & $-0,091$ & 0,109 & $-0,837$ \\
CP3 & 0,589 & 90,679 & 0,019 & $-0,075$ & $-0,107$ & $-0,030$ & 0,338 & $-0,409$ & $\underline{0,597}$ & $-0,306$ & $-0,080$ & 0,009 & 0,493 \\
CP4 & 0,468 & 94,932 & $-0,110$ & $-0,369$ & $-0,416$ & $-0,268$ & 0,338 & 0,162 & $-0,109$ & 0,068 & 0,214 & $\underline{-0,634}$ & $-0,035$ \\
CP5 & 0,204 & 96,783 & 0,137 & $-0,025$ & $-0,112$ & $-0,013$ & 0,335 & $\underline{0,544}$ & $-0,206$ & $-0,524$ & 0,204 & 0,427 & 0,137 \\
CP6 & 0,164 & 98,275 & $-0,005$ & $-0,196$ & $-0,235$ & $-0,137$ & 0,202 & $-0,071$ & 0,078 & $\underline{0,666}$ & 0,268 & 0,562 & 0,066 \\
CP7 & 0,135 & 99,503 & 0,045 & $-0,085$ & $-0,044$ & $-0,039$ & $-0,469$ & $-0,151$ & 0,230 & $-0,252$ & $\underline{0,780}$ & 0,003 & $-0,138$ \\
CP8 & 0,032 & 99,793 & 0,551 & $-0,156$ & $-0,367$ & $-0,094$ & $-0,400$ & 0,358 & 0,358 & 0,069 & $-0,329$ & $-0,012$ & $-0,001$ \\
CP9 & 0,016 & 99,938 & $\underline{-0,677}$ & 0,220 & 0,031 & $-0,231$ & $-0,135$ & 0,470 & 0,439 & 0,054 & $-0,044$ & 0,029 & 0,029 \\
CP10 & 0,006 & 99,997 & $-0,157$ & $\underline{-0,780}$ & 0,390 & 0,403 & $-0,050$ & 0,171 & 0,123 & 0,007 & $-0,062$ & 0,030 & 0,018 \\
CP11 & 0,000 & 100,000 & 0,246 & $-0,141$ & 0,592 & $-0,752$ & 0,054 & 0,011 & 0,016 & 0,001 & $-0,003$ & $-0,004$ & 0,005 \\
\hline
\end{tabular}

A- valor propio; AV - varianza acumulada (AV, en \%); G - germinación; IVG - índice de velocidad de germinación; VP - valor pico; VG - valor germinativo; E - índice de sincronización de la germinación; PN - plántulas normales; PA - plántulas anormales; GTS - ensayo de inmersión; GEA - envejecimiento acelerado; CE - conductividad eléctrica; $\mathrm{CP}$-longitud de plántulas. * - valores subrayados identifican los caracteres eliminados. 
minación, germinación, germinación tras el envejecimiento acelerado y la inmersión, plántulas normales, conductividad eléctrica y plántulas anormales, porque presentaron los mayores coeficientes de ponderación en los componentes con valores propios inferiores a 0,70 (cuadro 3 ). Mientras que, se apuntó a las variables valor pico, índice de sincronización y longitud de plántulas (apenas $27 \%$ de las variables) como portadoras de mayor variabilidad $y$, por tanto, más influyentes para determinar la diversidad entre los 25 árboles madre estudiados.

\section{DISCUSIÓN}

A partir de las diferencias entre las medias presentadas en todas las características evaluadas, se puede inferir que la variabilidad genética entre los árboles madre es de grande magnitud. Resultados semejantes fueron observados en otros estudios sobre variabilidad genética entre árboles a partir de calidad fisiológica de semillas (Santos et al. 2009, Roveri Neto y Paula 2017). Por otro lado, Tonetto et al. (2015) determinaron coeficientes de variación similares para la germinación y el índice de velocidad de germinación de H. heptaphyllus. Estos valores evidenciaron buena precisión de los experimentos, para evaluar todas las características.

La composición de los grupos de medias para el índice de velocidad de germinación, se aproxima bastante al observado para porcentaje de germinación, demostrando la eficiencia del mismo para caracterizar la calidad fisiológica de las semillas y para discriminar los genotipos. Marcos Filho (2005) señala que las condiciones de temperatura y humedad relativa son los factores ambientales más influyentes para el deterioro de las semillas. Se evidencia que el envejecimiento acelerado, provoca una pequeña reducción sobre la germinación, en relación al ensayo de germinación de semillas no envejecidas, pero, por otro lado, consigue separar los árboles en un mayor número de grupos, comparativamente a este, lo que facilita la selección de los mismos. Durante la inmersión en agua, las semillas permanecen en un ambiente anaeróbico cerrado, donde grandes cantidades de etanol son excretados por las semillas y provocan reducción del poder germinativo (Neumann et al. 1999). El ensayo de inmersión disminuye significativamente la germinación, demostrando ser más drástico en cuanto al grado de estrés que es sufrido por las semillas, en relación al envejecimiento acelerado, sin embargo, proporciona la formación del mismo número de grupos que el ensayo de germinación. Según Santos et al. (2009), al ser sometidas a estrés, gran parte de las semillas de bajo vigor, de cada árbol madre, son afectadas. Por el contrario, en condiciones óptimas para la germinación, las semillas de alto, medio y bajo vigor, de un mismo árbol, consiguen germinar. Por otro lado, dada la formación de un mayor número de grupos de árboles madre, a partir de la determinación del valor pico de la germinación y de la conductividad eléctrica de la solución de inmersión de las semillas, se evidencia que estas características tienen mayor influencia en la descripción de la variabilidad, permitiendo una mejor separación y selección de árboles productores de semillas con las características deseadas, en relación a los caracteres estudiados. La conductividad eléctrica de las semillas, posibilita la formación de varios grupos de árboles madre, contribuyendo, de ese modo, al análisis de la variabilidad genética; sin embargo, no siempre presenta la relación esperada con la germinación. Este hecho ya fue relatado por otros autores que también trabajaron con semillas de especies forestales (Santos et al. 2009, Roveri Neto y Paula 2017). Uno de los inconvenientes de este ensayo, en relación a los estudios de calidad fisiológica de semillas forestales, es exactamente, la presencia de variabilidad genética en los lotes de semillas, principalmente, cuando se trata de especies sin domesticación o mejoramiento (Bonner 1998). Justamente, este trabajo no pretendía evaluar la calidad fisiológica por medio del ensayo de conductividad eléctrica, sino acceder a la variabilidad existente entre los árboles madre. Al respecto, Bonner (1998) menciona que, para las semillas forestales, el ensayo de conductividad eléctrica difícilmente alcanza la misma precisión verificada con semillas agrícolas, ya que, en estas últimas, existe una mayor uniformidad genética dentro de cada lote.

Los genotipos más indicados para comenzar un programa de mejoramiento son los que presentan mayor distancia genética, sin embargo, su recomendación debe ser realizada, solamente, después de un profundo análisis de sus desempeños, en relación a los caracteres de interés (Cruz et al. 2004). La obtención de una medida de divergencia genética antes que los cruzamientos sean efectuados, permite al mejorador de plantas, concentrar sus esfuerzos en aquellas combinaciones que presentan mayor probabilidad de éxito. Para lograrlo, se busca trabajar con una populación-base compuesta por genotipos que presenten variabilidad genética, y a la vez, valores con medias altas, para el carácter que se pretende seleccionar (Cruz et al. 2011). Para los caracteres, germinación, índice de velocidad de germinación, valor germinativo, plántulas normales, conductividad eléctrica, germinación tras el envejecimiento acelerado y la inmersión, los árboles madre 3 y 5 originan semillas de mejor calidad fisiológica en relación a los demás. Por otro lado, los árboles 5 y 18 están localizadas en conjuntos diferentes, además presentan la mayor distancia genética (datos no presentados), demostrando así, mucha divergencia entre sí.

Los resultados del análisis de correlaciones confirmam que los árboles con buen desempeño germinativo en el ensayo de germinación, evidencian mantener, en grande parte, este desempeño en los ensayos de envejecimiento acelerado y de inmersión.

La distribución de los 25 árboles madre en el plano bidimensional, a partir del análisis de componentes principales, evidencia las semejanzas y diferencias entre los mismos, en base a los 11 caracteres evaluados, y se muestra bastante coherente con los agrupamientos del método de 
Ward. Oliveira (2010) al estudiar la variabilidad genética de caracteres morfológicos y de germinación en Tabebuia caraiba Bureau con la técnica de componentes principales, resalta que, cuanto más periférica es la posición de un árbol madre, su calidad específica aumenta, y que los valores extremos identifican aquella variabilidad que requiere de mayor investigación.

De ese modo, en base a los resultados de los agrupamientos de los árboles madre, se sugiere que para la recolección de semillas se prefieran árboles madre pertenecientes a grupos distintos, pues estos posibilitan la retención de variabilidad genética para el conjunto de caracteres evaluados. Por otro lado, semillas recolectadas de árboles madre pertenecientes a un mismo grupo, pueden, en un principio, constituir un mismo lote de semillas, ya que estas presentan semejanzas en cuanto a su calidad. Estas sugerencias pueden ser aplicadas para futuros trabajos, involucrando a los mismos árboles madre, por ejemplo, para la formación de ensayos de descendencia, visando a la conservación o mejoramiento de la especie.

La importancia o la contribución relativa de cada carácter para el estudio de la diversidad genética, evaluada a partir de los coeficientes asociados a los vectores propios, por la técnica de los componentes principales, tiene como principio el hecho de que la importancia o varianza de los componentes principales decrece desde el primero hasta el último componente estimado (Cruz et al. 2004). Por consiguiente, según estos autores, los últimos componentes son los responsables de explicar una mínima parte de la varianza total. De esta forma, es común descartar el carácter de mayor coeficiente de ponderación (elementos de vectores propios) a partir del último componente, hasta aquel cuyo valor propio no exceda 0,70 .

También, Cruz et al. (2004) consideran que por lo menos $80 \%$ de la variación total debe ser retenida por los dos primeros componentes principales, y en este estudio esto fue alcanzado, ya que los dos primeros componentes explican el $85,3 \%$ de la variabilidad total contenida en los datos. Para los mismos autores, los caracteres dispensables en los estudios de diversidad genética son aquellos invariantes y/o redundantes por presentar correlación con otros caracteres. Las estimativas de los coeficientes de correlación evidencian estas afirmativas. Así, el valor pico presenta altas correlaciones $(r>0,70)$ con todos los caracteres eliminados; también, se evidencian altas estimativas de correlación entre el índice de sincronización y el porcentaje de plántulas anormales $(\mathrm{r}=0,857)$. Por lo tanto, se verifica que estos pares están involucrando un carácter importante para la divergencia, de acuerdo con la técnica de los componentes principales. El valor pico representa a todos los caracteres que fueron descartados por ser redundantes. La longitud de plántulas muestra correlaciones bajas con todos los caracteres, razón por la cual, no es eliminada y permanece para explicar la divergencia. El índice de sincronización, al contrario de los demás caracteres que fueron descartados, muestra una correlación moderada con el valor pico $(r=0,672)$. Vale resaltar que $73 \%$ de los caracteres pueden ser excluidos, por no auxiliar en la discriminación de la diversidad genética. El principal interés en utilizar la técnica de los componentes principales reside en la posibilidad de descartar aquellos caracteres que no contribuyen para la discriminación del material evaluado, de este modo, mano de obra, tiempo y costos son reducidos (Cruz et al. 2004), facilitando la definición de estrategias para estudios similares con esta especie.

\section{CONCLUSIONES}

Las evidencias del estudio confirman la diversidad genética entre los árboles de $H$. heptaphyllus. Los ensayos de germinación, envejecimiento acelerado, conductividad eléctrica e inmersión evidencian variabilidad en la calidad fisiológica de semillas.

De los 11 caracteres de calidad fisiológica de semillas, evaluados mediante el análisis de componentes principales, ocho pueden ser eliminados. El valor pico, el índice de sincronización y la longitud de plántulas contribuyen efectivamente para la discriminación de los árboles madre.

La diversidad genética encontrada entre los árboles de H. heptaphyllus, permite orientar colectas de semillas de estos mismos árboles, para fines de conservación o mejoramiento de esta especie.

\section{AGRADECIMIENTOS}

Los autores expresamos nuestro total agradecimiento al personal de apoyo técnico y estudiantes de la Universidad Estadual Paulista, Facultad de Ciencias Agrarias y Veterinarias (UNESP-FCAV) que colaboraron con el trabajo de campo y laboratorio. También agradecemos el apoyo financiero de la Coordinación de Perfeccionamiento de Personas de Nivel Superior (CAPES) y del Consejo Nacional de Desarrollo Científico y Tecnológico (CNPq).

\section{REFERENCIAS}

Bonner FT. 1998. Testing tree seeds for vigor: a review. Seed Technology 20(1): 5-17.

Brasil. Ministério da Agricultura, Pecuária e Abastecimento. 2009. Regras para análise de sementes. Brasília, Brasil. MAPA/ACS. 399 p.

Broadhurst LM, T North, AG Young. 2006. Should we be more critical of remnant seed sources being used for revegetation? Ecological Management \& Restoration 7(3): 211-217.

Carvalho PER. 2003. Espécies arbóreas brasileiras. Brasília, Brasil. Embrapa Informação Tecnológica. p. 567-572.

CNCFlora (Base de Dados do Centro Nacional de Conservação da Flora, BR). 2012. Consultado 20 jul. 2016. Disponible en http://www.cncflora.jbrj.gov.br/portal/ptbr/profile/Handroanthus\%20heptaphyllus.

Cruz CD. 2001. Programa genes: Versão Windows: aplicativo computacional em genética e estatística. Viçosa, Brasil. UFV. 648 p. 
Cruz CD, AJ Regazzi, PCS Carneiro. 2004. Modelos biométricos aplicados ao melhoramento genético. Viçosa, Brasil. UFV. $480 \mathrm{p}$.

Cruz CD, FM Ferreira, AL Pessoni. 2011. Biometria aplicada ao estudo da diversidade genética. Visconde do Rio Branco, Minas Gerais, Brasil. Suprema. 620 p.

Czabator FJ. 1962. Germination value: an index combining speed and completeness of pine seed germination. Forest Science 8(4): 386-396.

Gentry AH. 1992. Bignoniaceae-Part II (Tribe Tecomeae). Flora Neotropica 25 (II): 1-370.

Giudice-Neto JD, AM Sebbenn, PY Kageyama. 2005. Genetic diversity of ex situ populations of Caesalpinia echinata Lam. Scientia Forestalis (69): 125-133.

Grings M, P Brack. 2011. Handroanthus heptaphyllus (ipê-roxo). In Coradin L, A Siminski, A Reis eds. Espécies nativas da flora brasileira de valor econômico atual ou potencial: plantas para o futuro - Região sul. Brasília, Brasil. MMA. p. 461-464.

Labouriau LG. 1983. A germinação das sementes. Washington, USA. OEA. 174 p.

Maguire JD. 1962. Speed of germination: aid in selection and evaluation for seedling emergence and vigour. Crop Science 2(2): 176-177.

Marcos Filho J. 1999. Testes de vigor: importância e utilização. In Krzyzanowski FC, RD Vieira, JB França Neto eds. Vigor de sementes: conceitos e testes. Londrina, Brasil. Abrates. p. 1-21.

Marcos Filho J. 2005. Fisiologia de sementes de plantas cultivadas. Piracicaba, Brasil. FEALQ. 495 p.

Martins SV. 2012. Restauração ecológica de ecossistemas degradados. Visçosa, Brasil. UFV. 293 p.
Mori NT, ES Mori, EV Tambarussi, MLT Moraes, Sebbenn AM. 2015. Sistema de cruzamento em populações de Handroanthus heptaphyllus (Vell.) Mattos e suas implicações para a coleta de sementes para fins de conservação e melhoramento genético. Scientia Forestalis 43(107): 675-681.

Neumann G, M Preissler, HA Azaizeh, V Romheld. 1999. Thiamine (Vitamin B1) deficiency in germinating seeds of Phaseolus vulgaris L. exposed to soaking injury. Journal of Plant Nutrition and Soil Science 162(3): 295-300.

Oliveira LZ. 2010. Variabilidade genética de caracteres morfológicos e germinação de Tabebuia caraiba (Mart.) Bur. (Bignoniaceae) no Município de Macapá, AP. Tesis de Maestría en Agronomia (Genética y Mejoramiento de Plantas). Jaboticabal, Brasil. Facultad de Ciencias Agrarias e Veterinarias, Universidad Estadual Paulista. 98 p.

Ribeiro RA, FM Rodrigues. 2006. Genética da conservação em espécies vegetais do cerrado. Revista de Ciências Médicas e Biológicas 5(3): 253-260.

Roveri Neto A, RC Paula. 2017. Variabilidade entre árvores matrizes de Ceiba speciosa St. Hil para características de frutos e sementes. Revista Ciência Agronômica 48(2): 318-327.

Santos FS, RC Paula, DZ Sabonaro, J Valadares. 2009. Biometria e qualidade fisiológica de sementes de diferentes matrizes de Tabebuia chrysotricha (Mart. Ex A. DC.) Standl. Scientia Forestalis 37(82): 163-173.

STATISTIC. 2011. Data Analysis Software System, version 10 (Software Statistic). Consultado 2016. Disponible en http:// www.statsoft.com

Tonetto T, MM Araujo, MFB Muniz, C Walker, ALP Berghetti. 2015. Storage and germination of seeds of Handroanthus heptaphyllus (Mart.) Mattos. Journal of Seed Science 37(1): 40-46. 